\title{
Functional characterization of AIBp, a novel Aurora-A binding protein in centrosome structure and spindle formation
}

\author{
ANN-SHUNG LIEU ${ }^{1,5^{*}}$, TAI-SHAN CHENG ${ }^{2 *}$, CHIA-HUA CHOU ${ }^{2,7^{*}}$, CHIA-HUNG WU $^{2}$, CHIA-YI HSU ${ }^{1,2}$, \\ CHI-YING F. HUANG ${ }^{3}$, LI-KWAN CHANG ${ }^{4}$, JOON-KHIM LOH ${ }^{5}$, CHUNG-SHING CHANG ${ }^{6}$, \\ CHING-MEI HSU ${ }^{7}$, SHEN-LONG HOWNG ${ }^{1,5}$ and YI-REN HONG ${ }^{1,2,8}$
}

\begin{abstract}
${ }^{1}$ Graduate Institute of Medicine, College of Medicine, ${ }^{2}$ Department of Biochemistry, Faculty of Medicine, College of Medicine, Kaohsiung Medical University, Kaohsiung; ${ }^{3}$ Institute of Clinical Medicine, National Yang-Ming University;

${ }^{4}$ Institute of Microbiology and Biochemistry, Department of Biochemical Science and Technology, National Taiwan University, Taipei; Department of ${ }^{5}$ Neurosurgery and ${ }^{6}$ Department of Dermatology, Kaohsiung Medical University Hospital; ${ }^{7}$ Department of Biological Sciences, National Sun Yat-Sen University; ${ }^{8}$ Center of Excellence for Environmental Medicine, Kaohsiung Medical University, Kaohsiung, Taiwan, R.O.C.
\end{abstract}

Received April 14,2010; Accepted May 28, 2010

DOI: 10.3892/ijo_00000691

\begin{abstract}
Aurora-A is involved in chromosome alignment, centrosome maturation, mitotic spindle assembly and regards to an oncogene. Aurora-A is also known to bind to several other proteins affecting its up-regulation or down-regulation and localization. However, how these different binding signals work together to regulate Aurora-A is not properly known. To explore more Aurora-A interacting proteins, the low-copy yeast two-hybrid screening using Aurora-A as bait protein was performed. One novel gene, AIBp, was demonstrated to associate with Aurora-A by the yeast two-hybrid method and in vitro GST pull-down assay. Molecular characterization showed that AIBp possessed a binding site at the C-terminal with Aurora-A (kinase domain). Interestingly, AIBp also interacts with hNinein at the $\mathrm{N}$-terminal, which overlaps with a previously reported hNinein and GSK3ß binding site. Using a kinase assay, AIBp interacts with the Aurora-A kinase domain functions as a positive regulator, whereas AIBp binding to hNinein appears to block the phosphorylation of hNinein by both Aurora-A and GSK3B. siRNA-mediated elimination of AIBp from HeLa cells, results in a doughnutlike shape, asymmetrical spindle pole and multiple spindle pole formation. We also demonstrated that both AIBp and
\end{abstract}

Correspondence to: Dr Yi-Ren Hong, Department of Biochemistry, Faculty of Medicine, College of Medicine, Kaohsiung Medical University, No. 100, Shih-Chuan 1st Road, Kaohsiung 80708, Taiwan, R.O.C.

E-mail: m835016@cc.kmu.edu.tw

${ }^{*}$ Contributed equally

Key words: Aurora-A, AIBp, ninein, centrosome, spindle, brain tumor
Aurora-A are co-overexpressed in various brain tumors. These studies demonstrate that AIBp may not only be required for the dynamic movement of Aurora-A at the centrosomes and spindle apparatus during the cell cycle, but may also be important during brain tumorigenesis.

\section{Introduction}

The faithful segregation of chromosomes involves the sequential activation of several protein kinases that are required for all or a subset of these mitotic events. The mitotic kinases include cyclin-dependent kinase 1 (CDK1/P34 ${ }^{\mathrm{CDC} 2}$ ) (1) pololike kinases (Plk) (2), NIMA-related kinases (Nek2), WARTS/ LATS1-related kinases and Aurora/Ipl1-related kinases $(3,4)$. Humans have three classes of Aurora kinases: Aurora-A, Aurora-B and Aurora-C (4). Aurora-A is known to bind to several other proteins which either up-regulate or downregulate Aurora-A activity, and may regulate localization of Aurora-A throughout the cell cycle. Activation of Aurora-A by TPX2 is required for bipolar spindle assembly $(5,6)$, whereas activation of Aurora-A by Ajuba and Bora are essential for mitotic entry (7-10).

Previously, we identified hNinein as a GSK3ß binding protein (11). We also identified a novel hNinein protein, CGI-99, that blocks hNinein phosphorylation by GSK3ß (12). More recently, Aurora-A regulation of GSK-3ß has been reported in gastric cancer cells (13). The discovery of several novel Aurora-A binding proteins and substrates has implicated Aurora-A in centrosomal maturation and separation, spindle assembly, kinetochore function and cell fate determination. However, how these different signals (substrates, co-factors and kinases) are integrated to regulate Aurora-A is not properly known.

To explore Aurora-A interacting proteins, we performed yeast two-hybrid screening using full-length Aurora-A (1-403 aa) as a bait protein. AIBp (AIK binding protein) was demonstrated to associate with Aurora-A in vitro and in vivo. 
We also showed that AIBp anchors to both Aurora-A and hNinein to prevent the phosphorylation of hNinein by both Aurora-A and GSK3B. Moreover, AIBp and Aurora-A are highly co-expressed in various brain tumors. Our data suggest that AIBp may not only participate in the centrosome architecture, but also contributes to brain tumorigenesis.

\section{Materials and methods}

Cloning and DNA sequencing. Aurora-A, Aurora-A kinase dead (KD), Aurora-B and Aurora-C (from Dr T.K. Tang, Academia Sinica, Taiwan) were cloned into pBGT9 (Clontech). AIBp fragments were amplified from a testis cDNA library by PCR and cloned into pACT2 (Clontech), then completely sequenced. Plasmid pET32a-AIBp, which expresses Histagged AIBp in E. coli BL21/(DE3), was constructed by inserting AIBp DNA fragments into pET-32a (Novagen). Plasmid pGEX-KG-Aurora-A, which expresses GST-tagged Aurora-A in E. coli BL21/(DE3), was constructed by inserting Aurora-A DNA into pGEX-KG. Plasmid pcDNA-AIBp, which expresses HA-tagged AIBp, was amplified by PCR and cloned into the vector pcDNA. Plasmid p Flag-CMVAurora-A, which expresses FLAG-tagged Aurora-A, was constructed by inserting a PCR-amplified Aurora-A DNA fragment into pFlag-CMV. The nucleotide sequencing was performed by ABI PRISM ${ }^{\text {TM }} 3730$ Genetic Analyzer (PerkinElmer).

Yeast hybrid techniques. Standard techniques were used for yeast two-hybrid screening $(14,15)$. Briefly, the Aurora-A gene was cloned in frame with the GAL4 DNA binding domain (GAL4 BD) in the pBGT9 vector (Clontech) to yield pBGT9-Aurora-A. A human testis cDNA library (Clontech) was screened by co-transforming yeast YRG-2 (Stratagene) with the pBGT9-Aurora-A bait plasmid DNA. Positive clones were selected based on the ability of the cells to grow on Trp, Leu and His dropout media supplemented with 3-aminotriazole (3-AT) together with a blue colony color by ß-galactosidase filter assay. The three-hybrid system of interactions between reporter yeast cells harboring the pBridge (Clontech) and pGAD vectors containing Aurora-A, AIBp (as a bridging protein) and hNinein were tested for the His ${ }^{+}$ phenotype.

Cell culture, transfections, synchronization, RNA interference and indirect immunofluorescence. HeLa cells were cultured and transient transfection studies were carried out as previously described (16). For indirect immunofluorescence studies, the cells were probed with rabbit anti-Aurora-A serum $(1: 500$, our preparation) or mouse anti-Aurora-A antibody $(1: 500 ; 35 \mathrm{C} 1$, Abcam), rabbit anti-AIBp antibody (1:250, our preparation), mouse anti- $\gamma$-tubulin antibody (1:1000; GTU-88, Sigma) and mouse anti- $\alpha$-tubulin antibody (1:1000; DM1A, Sigma). The secondary antibodies were Rhodamine-conjugated goat anti-rabbit or goat anti-mouse antibodies (1:500; Invitrogen) and FITC-conjugated goat anti-rabbit or anti-mouse antibodies (1:500; Invitrogen). DNA was stained with DAPI $(2 \mu \mathrm{g} / \mathrm{ml}$, Roche). For synchronization, cells were treated with $200 \mathrm{ng} / \mathrm{ml}$ nocodazole (Sigma). RNA interference mediated by duplexes of 25-nt
RNAs was performed on human HeLa cells as described previously $(17,18)$. The following siRNA duplex oligonucleotides were used. AIBp: sense, 5'-GAAGAGUGUUUC AUCUCAUACAGAA-3' and antisense 5'-UUCUGUAUGA GAUGAAACACUCUUC-3'. A scrambled siRNA duplex was used as the negative control (Invitrogen). Immunofluorescent cell images were acquired using an Olympus LSM Fluoview 500 confocal laser scanning microscope (Olympus). Images were processed with Ulead Photoimpact software.

Northern blot analysis. Pre-made blots containing poly-(A) RNA isolated from adult human tissues including the heart, brain, placenta, lung, liver, skeletal muscle, kidney, pancreas, spleen, thymus, prostate, testis, ovary, small intestine, colon and leukocyte were purchased from Clontech and used for hybridization with AIBp PCR-amplified specific probe (sense, 5'-CGGGATCCAAAGTTCTTGTTTGCTAGACC GAG-3'; anti-sense, 5'-CCGCTCGAGTCACCGCAGATGT TTCTGCCTCTT-3'). Blots were hybridized according to Church and Gilbert (19) with a 402-bp AIBp 3-end fragment labeled with $\left[\alpha^{-32} \mathrm{P}\right] \mathrm{dCTP}$.

$R T$-PCR. The cDNAs from human brain were obtained from Clontech and used as templates for tissue-specific PCR. Total RNA was extracted from brain tumors by the acid guanidinium phenol/chloroform method and cDNA was synthesized with moloney murine leukemia virus reverse transcriptase (Stratagene). In order to identify AIBp expression in different brain tumors, primers of AIBp (sense, 5'-AAAGTTCTT GTTTGCTAGACCGAG-3'; anti-sense, 5'-TCACCGCAG ATGTTTCTGCCTCTT-3') were used.

GST pull-down assay. pGEX-KG-Aurora-A (GST-Aurora-A), pET32a-hNinein C-terminal 1617-2090 aa (His-hNinein) and pET32a-AIBp (His-AIBp) were expressed in E. coli BL21/ (DE3). Then glutathione-agarose beads were added to the supernatant and the mixture was incubated under shaking for $1 \mathrm{~h}$ at $4^{\circ} \mathrm{C}$. The beads were washed 3 times with $1 \mathrm{X}$ TBS buffer. After washing, the beads were added to the purified protein $(50 \mu \mathrm{g})$ prepared from E. coli lysate containing Histagged proteins. The reaction mixture was incubated on ice for $1 \mathrm{~h}$ to allow binding between GST-Aurora-A protein and His-tagged AIBp proteins or GST-AIBp and His-tagged hNinein. Proteins were finally analyzed by SDS-polyacrylamide gel electrophoresis and immunoblotting as previously described (12).

Immunoprecipitation. pFlag-CMV-Aurora-A (Flag-Aurora-A), pcDNA-hNinein (HA-tagged hNinein) and pcDNA-AIBp (HA-tagged AIBp) were expressed in HeLa cells. HeLa cells were collected from one 10-cm dish in $1 \mathrm{ml}$ of mRIPA buffer. Lysate $(50 \mu 1)$ of the supernatant was removed for immunoblotting. The anti-Flag antibody (Roche) was added to the supernatant for $1 \mathrm{~h}$ at $4^{\circ} \mathrm{C}$. Protein-A/G-agarose beads $(30 \mu \mathrm{l})$ (Calbiochem) were added to the lysate, and the mixture was incubated with shaking for $1 \mathrm{~h}$ at $4^{\circ} \mathrm{C}$. Immunoprecipitates were washed three times with mRIPA buffer. Proteins binding to the beads were eluted by adding $20 \mu \mathrm{l}$ of $2 \mathrm{X}$ electrophoresis sample buffer and analyzed by immunoblotting with anti-HA antibody (Roche). 
A

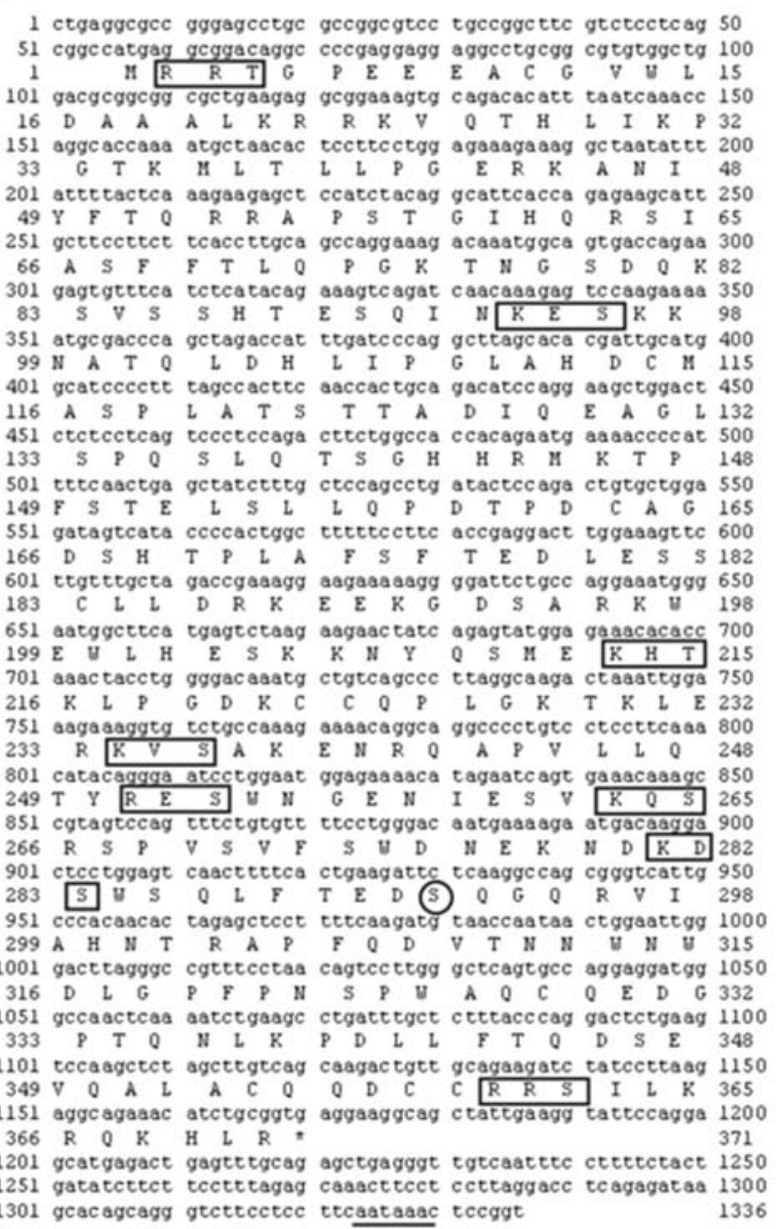

B

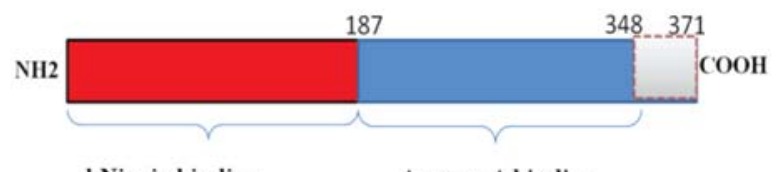

hNinein binding

Auroua-A binding

C

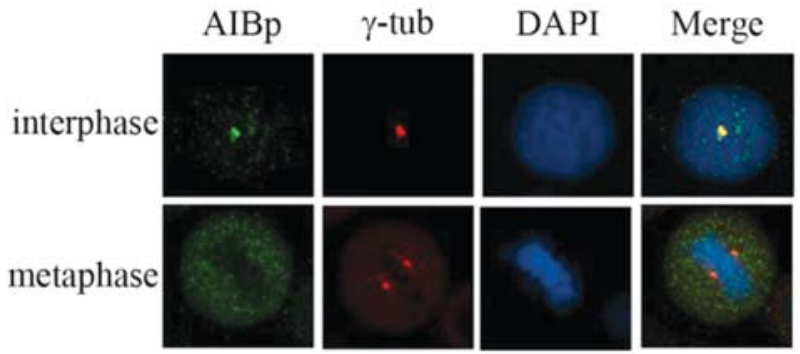

Figure 1. Identification and characterization of AIBp. (A) Nucleotide and deduced amino acid sequences of AIBp. Putative Aurora-A phosphorylation sites $(\mathrm{R} / \mathrm{K}-\mathrm{X}-\mathrm{S} / \mathrm{T})$ are boxed and the ATM phosphorylation site (D/E-S-Q) at S292 is marked with a circle. The AATAAA polyadenylation signal is underlined. The nucleotide sequences for AIBp have been assigned to the GenBank database as accession no. GQ844687. (B) The N-terminal region of AIBp (1-187 aa) represents a hNinein binding domain (red). The C-terminal region (180-348 aa) contains an Aurora-A binding domain (blue) and spliced variant of C1orf135 (gray). (C) Localization of AIBp. AIBp was localized to the centrosome in interphase (upper panel), and to the spindle pole in metaphase (lower panel). Note AIBp and $\gamma$-tubulin in a representative interphase or metaphase cell.

In vitro kinase assays. Kinase reaction was carried out as described (12). Briefly, the AIBp protein and hNinein protein were purified; myelin basic protein (MBP, Sigma) and histone H3 (Roche) incubated with Aurora-A or GSK3ß kinases in kinase buffer [100 mM HEPES (pH 7.4), $1 \mathrm{mM}$ dithiothreitol, $10 \%$ glycerol, $0.4 \mathrm{mM}$ ATP, $80 \mathrm{mM} \mathrm{MgCl}_{2}$ and $10 \mu \mathrm{Ci}$ of $\left[\gamma^{-}{ }^{32} \mathrm{P}\right] \mathrm{ATP}($ Amersham, $\left.3000 \mathrm{Ci} / \mathrm{mM})\right]$. The assays were carried out for $1 \mathrm{~h}$ at $30^{\circ} \mathrm{C}$. Reactions were stopped by the addition of $2 \mathrm{X}$ sample buffer and heated at $95^{\circ} \mathrm{C}$ for $5 \mathrm{~min}$, following by SDS-PAGE and detection by autoradiography.

\section{Results and Discussion}

Identification of AIBp as an Aurora-A binding protein. Since high level Aurora-A is toxic to yeast, the plasmid construct was made in yeast expression vector pGBT-9 for low-copy constitutive expression of the full-length Aurora-A. A novel human protein designated as AIBp was identified. The fulllength AIBp cDNA contains a 1116-bp open reading frame that encodes a 371-amino acid polypeptide with a predicted molecular weight of $41 \mathrm{kDa}$ (GenBank GQ844687) (Fig. 1A). Comparison of the protein and nucleotide sequences of AIBp with the GenBank database revealed that it was similar to an uncharacterized protein, C1orf135 (NM_024037), and it appears that AIBp possesses an alternative splicing isoform of C1orf135 (Fig. 1B). Localization of AIBp to the centrosome was confirmed by comparison with $\gamma$-tubulin. The data showed that AIBp is a centrosomal protein (Fig. 1C).

Expression of AIBp in various human tissues. By Northern blot analysis, a 2.4-kb transcript was found in heart and skeletal muscles, whereas 2.6 - and $2.0-\mathrm{kb}$ transcript variants were found in testis and placenta (Fig. 2A). In brain, however, the transcript was absent (Fig. 2A, lane 2). The AIBp expression level in adult tissues was further confirmed by RT-PCR in heart, placenta and testis except skeletal muscles (Fig. 2B). It should be noted that this protein is abundant in the testis.

AIBp interacts with Aurora-A and hNinein: AIBp as a bridging protein. To determine the interaction region involved between AIBp and Aurora-A, a yeast two-hybrid system was 
$\mathbf{A}$

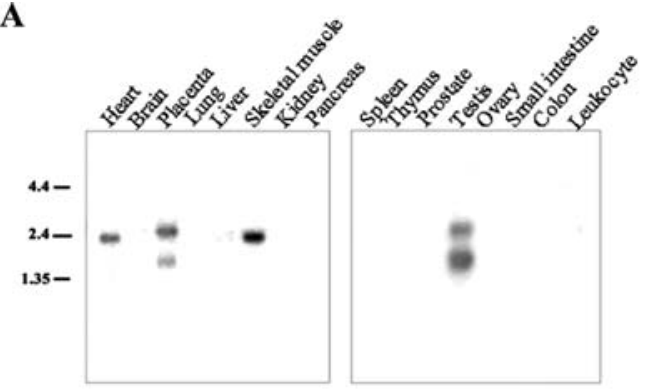

B

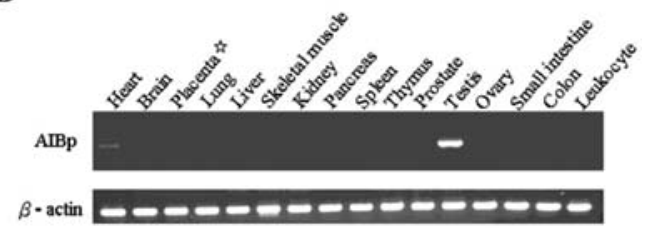

Figure 2. Northern blot and RT-PCR analysis of AIBp expression in various human tissues. (A) Northern blot analysis of adult tissues shows a 2.4-kb transcript in heart and skeletal muscles, whereas 2.6- and 2.0-kb transcript variants were found in placenta and testis. (B) Quantitative PCR analysis of AIBp expression in different human tissues. Only heart, placenta and testis tissue has been amplified. "रFaint band. used to test the interaction between Aurora-A (Fig. 3A) and AIBp. The data showed that the C-terminal of AIBp (187371 aa) specifically interacts with Aurora-A (Fig. 3B). It should be also noted that another splicing form also binds to Aurora-A (data not shown), indicating that some regions (349-371 aa, Fig. 1B) were not required for binding. We further mapped the region of this protein-protein interaction within Aurora-A. The data clearly showed that AIBp used C-terminus (187-371 aa) to associate with the C-terminus (133-403 aa) of the Aurora-A kinase domain, and kinase activity (not KD) and D-box domain were required for this interaction (Fig. 3C). We also demonstrated that proteinprotein interaction specifically only binds to Aurora-A, but not Aurora-B or Aurora-C (Fig. 3D). We therefore concluded that both the kinase domain and D-box are required for Aurora-A and AIBp interaction. Previously, we identified a centrosomal protein hNinein that interacts with GSK3ß (11). Interestingly, AIBp also interacts with hNinein at $\mathrm{N}$-terminal, which overlapped with previously reported hNinein and GSK3ß binding site. To further confirm these protein-protein interactions, AIBp and Aurora-A or hNinein were overexpressed for an in vitro binding assay. The results of in vitro

A

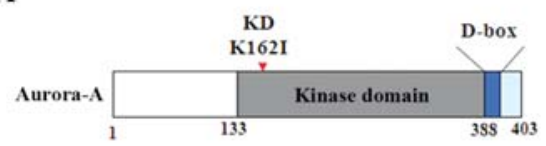

B

DNA-binding hybrid

Activation hybrid

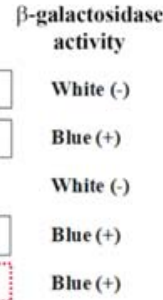

\begin{tabular}{|c|c|}
\hline GAL4 BD & \\
\hline GAL4BD & Aurora-A \\
\hline GAL4 BD & Aurora-A \\
\hline GAL 4 BD & Aurora-A \\
\hline GAL4 BD & Aurora-A \\
\hline
\end{tabular}

\begin{tabular}{|c|c|}
\hline GAL4AD & AIBp (1-371) \\
\hline GAL4AD & AIBp (1-371) \\
\hline GAL4AD & AIBp (1-186) \\
\hline GAL4AD & AIBp (1-348) \\
\hline GAL4AD &
\end{tabular}

Blue (+)

C

\begin{tabular}{l} 
DNA-binding hybrid \\
\begin{tabular}{|l|l|l|}
\hline GAL4 BD & & Aurora-A (1-403) \\
\hline GAL4 BD & & Aurora-A (1-132) \\
\hline GAL4 BD & & \\
\hline GAL4 BD & & Aurora-A (KD) \\
\hline GAL4 BD & Aurora-A(1-346) \\
\hline GAL4 BD & \\
\hline
\end{tabular} \\
\hline
\end{tabular}

\begin{tabular}{|c|c|c|}
\hline \multicolumn{2}{|c|}{ Activation hybrid } & $\beta$-galactosidase \\
\hline GALAAD & AIBp (1-371) & Blue (+) \\
\hline GALAAD & AIBp (1-371) & White (-) \\
\hline GALAAD & AIBp (1-371) & Blue (+) \\
\hline GALAAD & $\operatorname{AIBp}(1-371)$ & White (-) \\
\hline GALAAD & AIBp (1-371) & White (-) \\
\hline GAL4AD & $---A I B p($ & Blue (+) \\
\hline
\end{tabular}

D

\begin{tabular}{|c|c|}
\hline GAL 4 BD & Aurora-A \\
\hline GAL4 BD & Aurora-B \\
\hline GAL 4 BD & Aurora.C \\
\hline
\end{tabular}

\begin{tabular}{|c|c|c|}
\hline \multicolumn{2}{|c|}{ Activation hybrid } & $\beta$-galactosidase \\
\hline GAL 4 AD & AIBp (1-371) & Blue $(+)$ \\
\hline GAL 4 AD & AIBp (1-371) & White $(-)$ \\
\hline GAL4AD & $\operatorname{AIBp}(1-371)$ & White (-) \\
\hline
\end{tabular}

Figure 3. Yeast two-hybrid assay showing the interaction of AIBp and Aurora-A. (A) Schematic diagram of Aurora-A. (B) Map of the AIBp interaction with Aurora-A. (C) Map of the Aurora-A interaction with AIBp. ${ }^{+}$positive interaction; ${ }^{-}$negative interaction. (D) Comparisons of Aurora kinase family interaction with AIBp. 
A

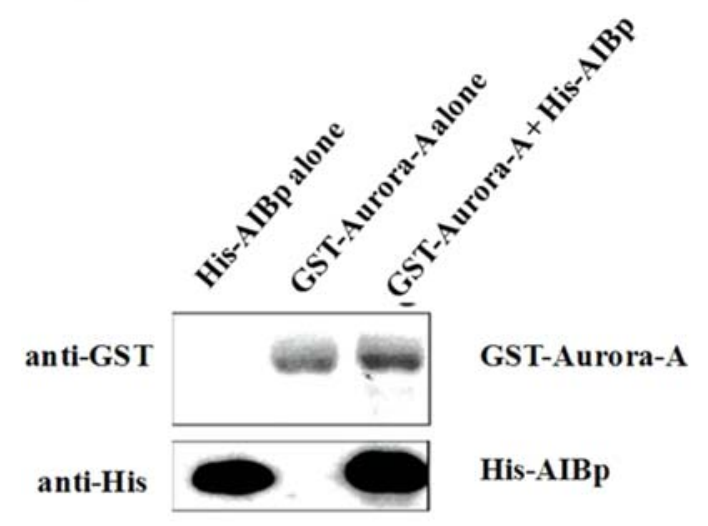

C

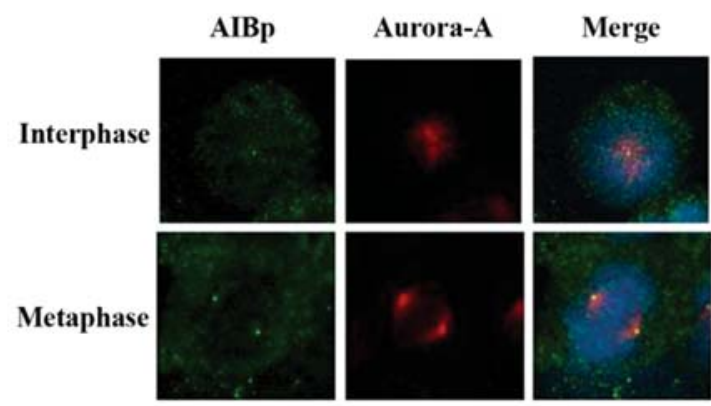

E

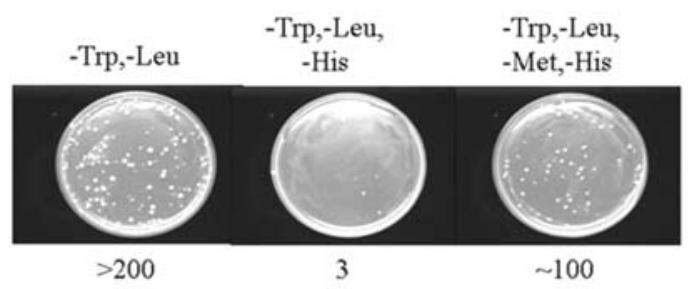

B

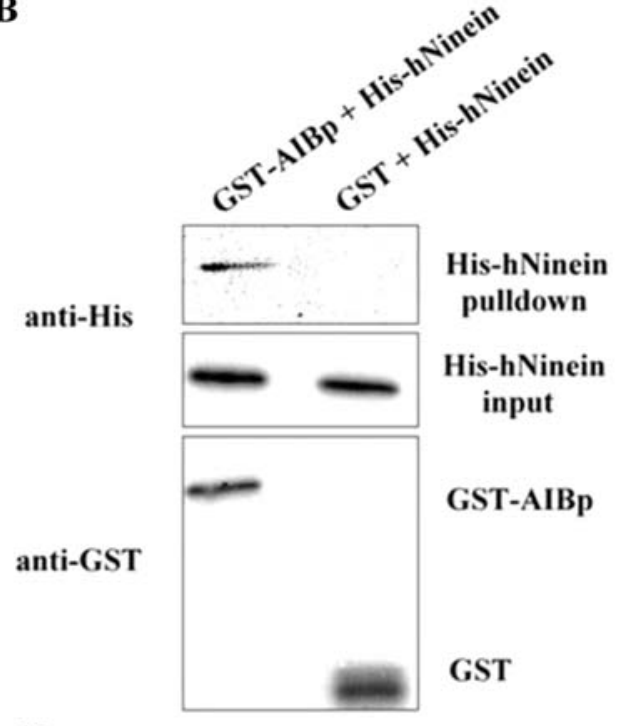

D

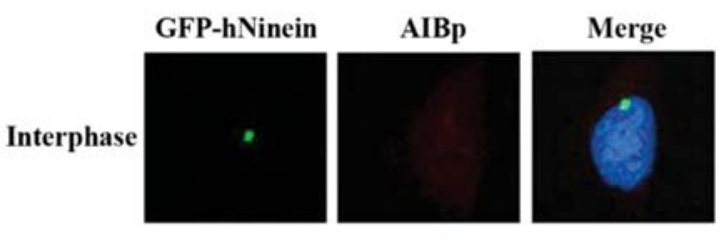

F

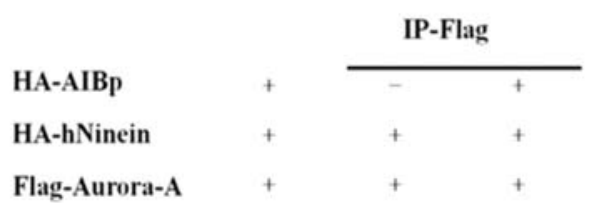

IB-HA

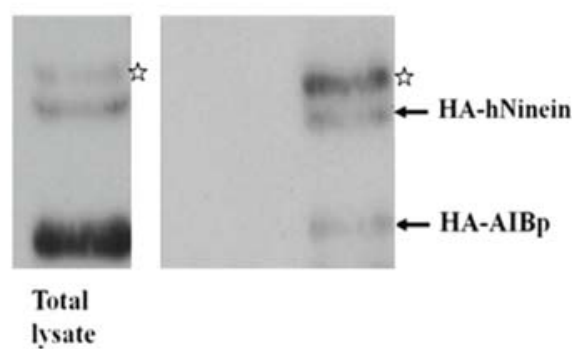

Figure 4. In vitro pull-down assays and molecular image analysis. (A) Interaction of AIBp and Aurora-A was analyzed in the presence (+) or absence (-) of assay mixtures containing purified GST-Aurora-A and His-tagged AIBp. (B) Interaction of AIBp and hNinein analyzed in the presence (+) or absence (-) of assay mixtures containing purified GST-AIBp and His-tagged C-terminal hNinein (1617-2090 aa). (C) Molecular image analysis indicated co-localization of AIBp and Aurora-A. (D) Co-localization of AIBp and hNinein. (E) Yeast three-three hybrid AIBp, Aurora-A and hNinein interact together. (F) Co-

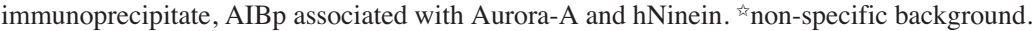

GST-pull-down assay showed that GST-Aurora-A binds to His-Tag-AIBp (Fig. 4A). To search for the binding partner of the $\mathrm{N}$-terminal region of AIBp, the $\mathrm{C}$-terminal region of hNinein (1617-2090 aa) was used in the yeast two-hybrid system (data not shown) with pull-down analysis (Fig. 4B), which indicated that hNinein indeed binds to AIBp. In addition, molecular image analysis showed co-localization of Aurora-A with AIBp and hNinein with AIBp (Fig. 4C and D, respectively). We then used the yeast three-hybrid system and co-IP to demonstrate that these three proteins interact together (Fig. 4E and F), suggesting that AIBp does indeed act as a bridging protein.
AIBp interaction with Aurora-A functions as a positive regulator. To examine whether AIBp is a substrate for Aurora-A, we performed an in vitro kinase assay. Our data showed that Aurora-A can phosphorylate AIBp (Fig. 5A) and MBP (Fig. 5B, bottom panel) To further explore the endogenous substrates involved in Aurora-A, the kinase assay also showed the phosphorylation of histone $\mathrm{H} 3$ (Fig. 5B, middle panel). Importantly, AIBp interaction with Aurora-A functions as a positive regulator on histone $\mathrm{H} 3$ and $\mathrm{MBP}$ (Fig. 5B).

AIBp interaction with hNinein blocks hNinein phosphorylation by both Aurora-A and GSK3 3 . The physiological 
A

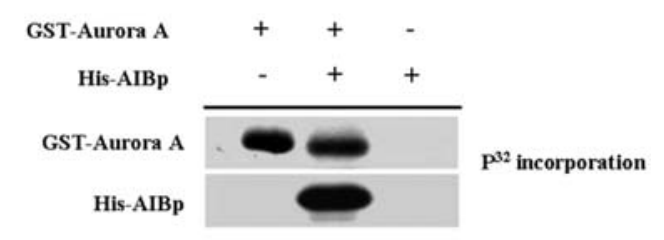

B

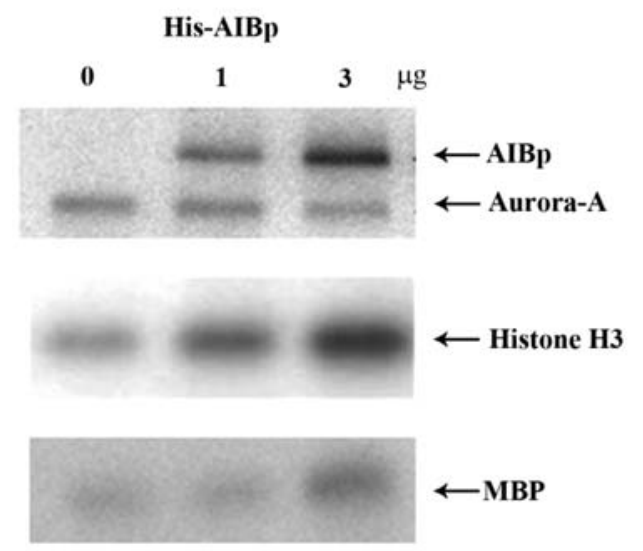

Figure 5. In vitro kinase assay. In vitro kinase assay was performed using purified GST-tagged Aurora-A and His-tagged AIBp. Assays were carried out using His-tagged AIBp as substrate incubated with GST-tagged Aurora-A in the present $\left[\gamma^{-32}\right.$ p]ATP. (A) Aurora-A phosphorylates AIBp. (B) Histone $\mathrm{H} 3$ and MBP. role of AIBp as a bridging protein of Aurora-A and hNinein remains unclear. We therefore performed a kinase assay to show the phosphorylation of two C-terminals of hNinein (1617-2090 aa and 2010-2090 aa) by Aurora-A (Fig. 6A, lanes 1 and 3) or GSK3ß (Fig. 6B, lanes 1 and 3). Our data showed that AIBp anchors and decreases hNinein phosphorylation by both Aurora-A and GSK3ß (Fig. 6A and B, lanes 2 and 4 compared to 1 and 3 ). It should be also noted that AIBp does act as a substrate for Aurora-A, but not for GSK3B (Fig. 6A and B, lanes 2 and 4), suggesting that AIBp may be involved with and restricted to hNinein phosphorylation during the cell cycle. It is noted that the region of hNinein binding to AIBp overlapped with the GSK3ß binding site (data not shown). These observations suggest that AIBp anchors and regulates hNinein, GSK $3 B$ and Aurora-A in a complex way. A similar scenario was observed with CGI-99, which blocks hNinein phosphorylation by GSK3ß (12). In addition, a recent paper indicated that C1orf135 (a spliced variant of AIBp) served as a substrate for ATM kinase at residue S292, suggesting that AIBp may have a broader role in the cell cycle (Fig. 1A) (20). It will be interesting to determine whether AIBp with its variant or CGI-99 truly regulates hNinein phosphorylation through GSK3B, Aurora-A and /or ATM kinases.

AIBp is required for localization of Aurora-A to spindle poles and spindle. Upon siRNA-mediated elimination of AIBp from HeLa cells for $48 \mathrm{~h}$ and followed by fixation and staining

B
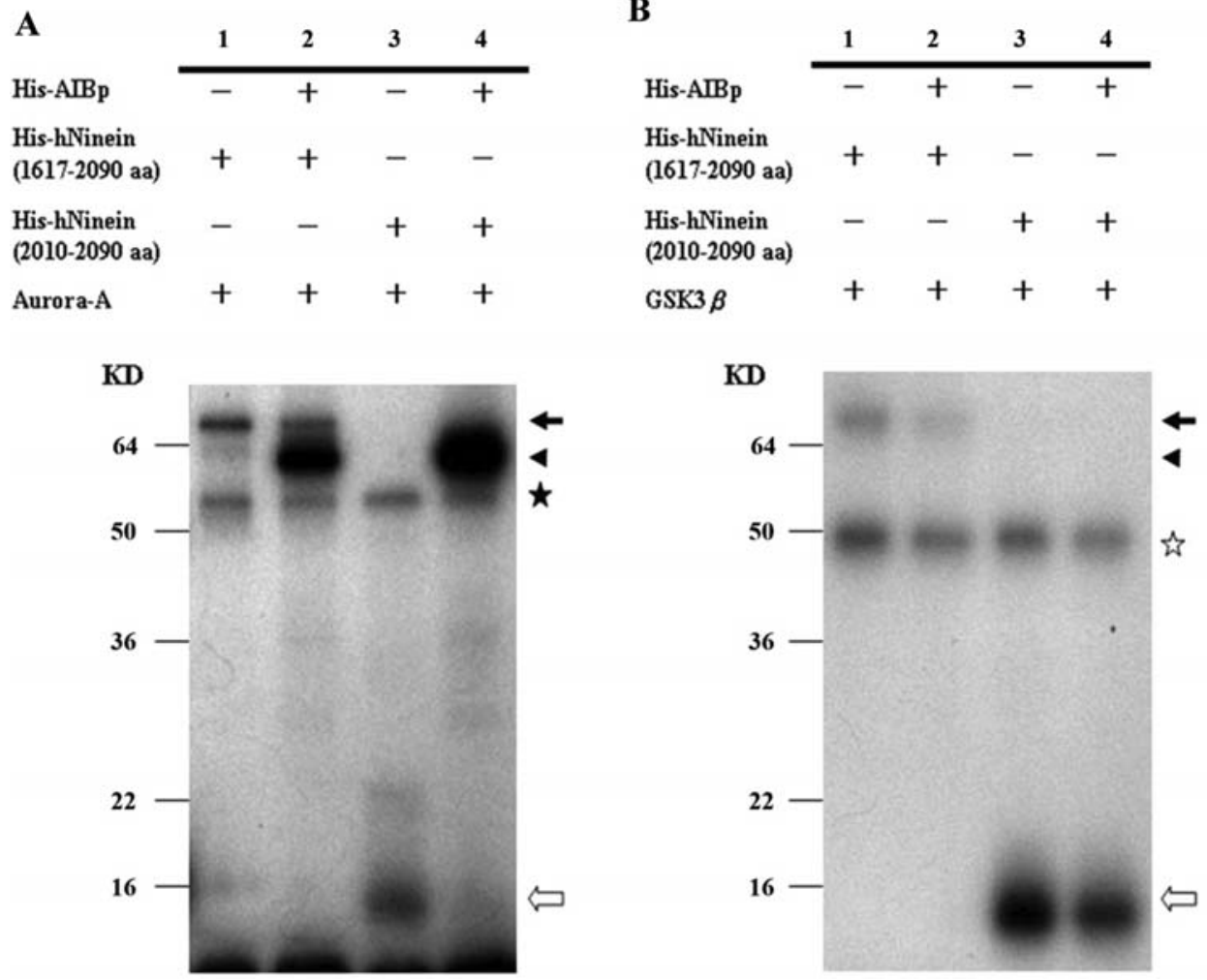

Figure 6. AIBp interaction with hNinein blocks hNinein phosphorylation by both Aurora-A and GSK3ß. Phosphorylation of two C-terminal fragments of hNinein by Aurora-A or GSK3ß. (A) The reactions were analyzed either in the presence (+) or absence (-) of assay mixtures containing purified His-taggedAIBp, His-tagged C-terminal hNinein (1617-2090 aa), His-tagged C-terminal hNinein (2010-2090 aa) and His-tagged Aurora-A. (B) Mixtures containing purified His-tagged-AIBp, His-tagged C-terminal hNinein (1617-2090 aa), His-tagged C-terminal hNinein (2010-2090 aa) and His-tagged-GSK3ß. Arrow indicates phosphorylated hNinein band (1617-2090 aa). Empty arrow indicates hNinein band (2010-2090 aa). Arrowhead indicates AIBp. ${ }^{\star}$ Aurora-A; GSK3ß autophosphorylation. 
$\mathbf{A}$
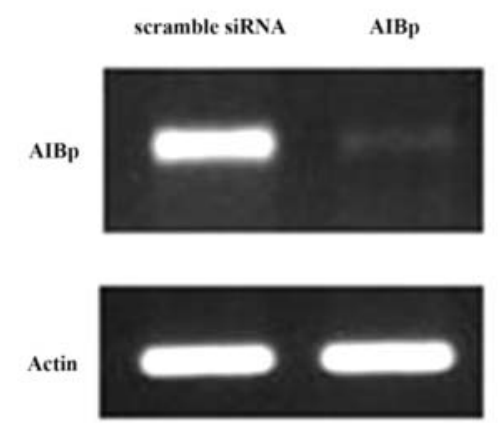

B

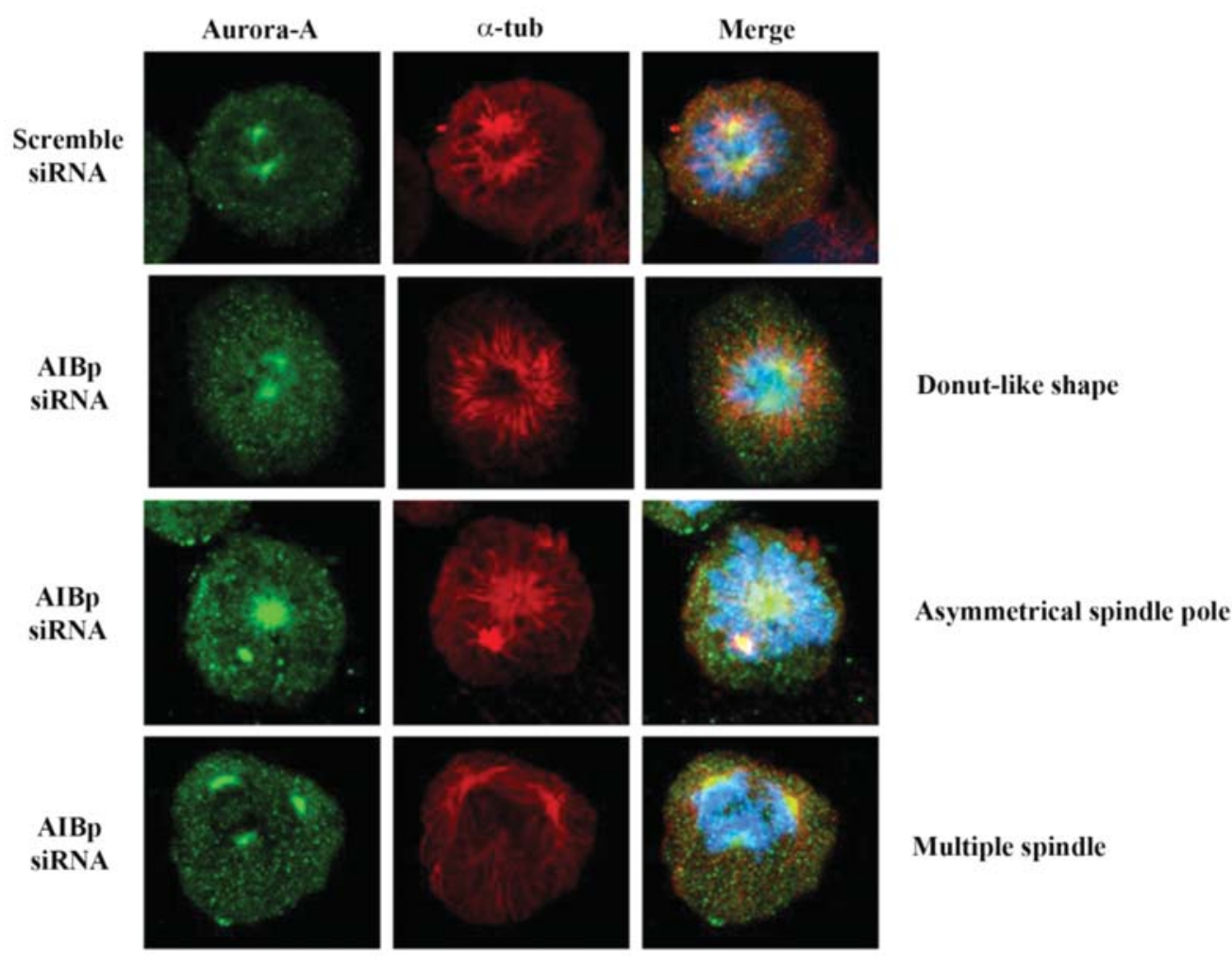

Figure 7. AIBp is required for recruiting Aurora-A to spindle and spindle poles. (A) RT-PCR analyses of total extracts form HeLa cells treated for $24 \mathrm{~h}$ with an AIBp-specific siRNA or a scramble siRNA. (B) HeLa cells were treated for $48 \mathrm{~h}$ with an AIBp-specific siRNA duplex and then fixed and stained with antibodies against Aurora-A (green) and $\alpha$-tublin (red). The two images are merged together with DAPI staining of DNA on the right showing doughnut-like shape, multiple spindle poles and asymmetrical spindle poles formation as indicated.

with antibodies against Aurora-A (green) and $\alpha$-tublin (red), results in doughnut-like shape $(36 \%)$, multiple spindle poles $(20 \%)$, and asymmetrical spindle pole formation $(28 \%)$ (Fig. 7). These data indicated that AIBp is required for recruiting Aurora-A to spindle pole and spindle. It is reported that TPX2 functions as a positive regulator and is also required for localization of Aurora-A to spindle poles and spindle (21).

AIBp and Aurora-A are co-expressed in brain tumors. To verify the data in Northern blot and RT-PCR in Fig. 2, we further examined the AIBp and Aurora-A expression profile in various brain tumors. In this study, 41 human brain tumor samples were examined by RT-PCR. The data showed that AIBp and Aurora-A were overexpressed in brain tumors (Fig. 8A), suggesting that aberrant expression of AIBp may contribute to the pathogenesis of brain tumors. Aurora-A and AIBp were $93 \%$ co-overexpressed in astrocytoma and $>50 \%$ in other brain tumors (Fig. 8B). It should also be noted that AIBp is dominantly expressed in the testis, but not in the normal brain (Fig. 2B), implying that this protein may act as a cancer-testis oncogene in brain tumorigenesis.

In conclusion, we have isolated a novel bridging protein AIBp, which may be involved in the functional regulation of hNinein by Aurora-A and GSK3ß in the centrosome structure. In addition, our data also suggest that AIBp to 


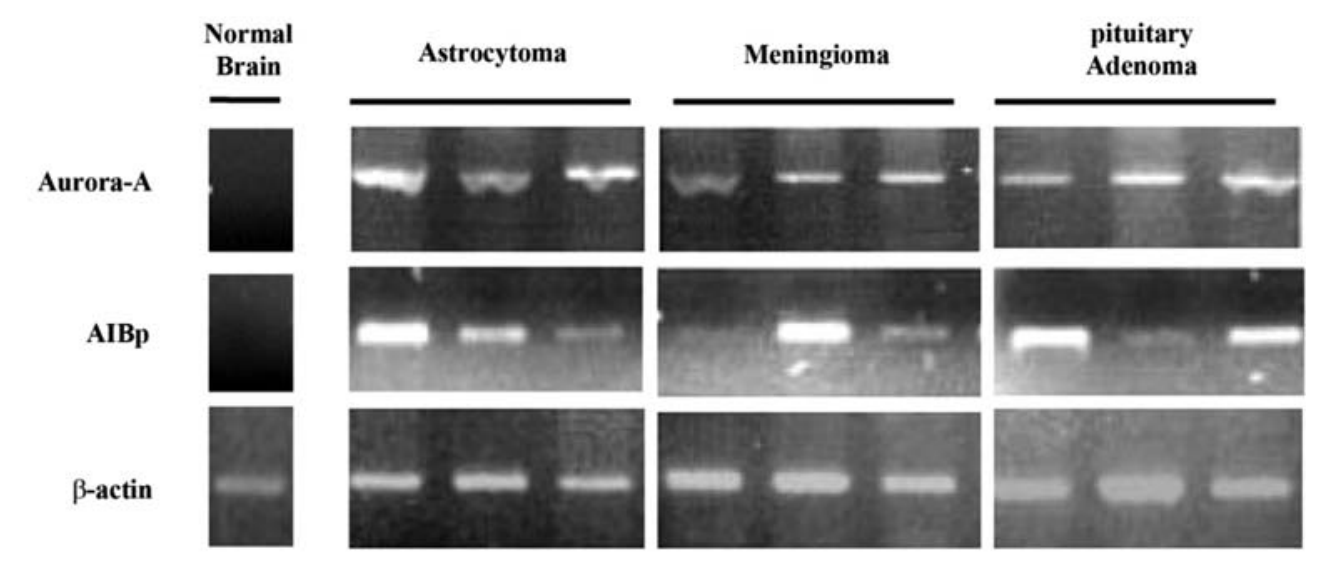

B

\begin{tabular}{lllll}
\hline Pathological Lesion & Astrocytoma & Meningioma & $\begin{array}{l}\text { Pituitary } \\
\text { adenoma }\end{array}$ & Total \\
\hline Aurora-A overexpression (\%) & $13 / 14(93)$ & $12 / 17(71)$ & $9 / 10(90)$ & $34 / 41(83)$ \\
AIBp overexpression (\%) & $13 / 14(93)$ & $9 / 17(53)$ & $5 / 10(50)$ & $27 / 41(66)$
\end{tabular}

Figure 8. AIBp and Aurora-A are expressed in brain tumors. (A) RT-PCR analysis of Aurora-A and AIBp in various brain tumors. Data shown are representative of at least three independent experiments. B-actin was used as internal control. (B) Distribution of Aurora-A and AIBp in various brain tumors.

Aurora-A and GSK3ß possesses distinct substrate specificity dependent on different stages of the cell cycle. Moreover, AIBp is required for localization of Aurora-A to spindle poles and spindle. We conclude that AIBp may be required for dynamic movement of Aurora-A at centrosomes and spindle apparatus during the cell cycle, and may also be important in brain tumorigenesis.

\section{Acknowledgements}

This work was supported by grants NSC97-2314-B-037-013 (Taiwan, R.O.C.) to S.L.H.; NSC96-2320-B-037-004, MOE98-U19609-6-3 and NHRI-EX98-9809SI to Y.R.H.

\section{References}

1. Morgan DO: Principles of CDK regulation. Nature 374: 131-134, 1995.

2. Lane HA and Nigg EA: Cell-cycle control: POLO-like kinases join the outer circle. Trends Cell Biol 7: 63-68, 1997.

3. Mayor T, Meraldi P, Stierhof YD, Nigg EA and Fry AM: Protein kinases in control of the centrosome cycle. FEBS Lett 452: 92-95,1999.

4. Nigg EA: Mitotic kinases as regulators of cell division and its checkpoints. Nat Rev Mol Cell Biol 2: 21-32, 2001.

5. Trieselmann N, Armstrong S, Rauw J and Wilde A: Ran modulates spindle assembly by regulating a subset of TPX2 and Kid activities including Aurora A activation. J Cell Sci 116: 4791-4798, 2003.

6. Tsai MY, Wiese C, Cao K, Martin O, Donovan P, Ruderman J, Prigent $C$ and Zheng Y: A Ran signalling pathway mediated by the mitotic kinase Aurora A in spindle assembly. Nat Cell Biol 5: 242-248, 2003.

7. Hirota T, Kunitoku N, Sasayama T, Marumoto T, Zhang D, Nitta M, Hatakeyama K and Saya H: Aurora-A and an interacting activator, the LIM protein Ajuba, are required for mitotic commitment in human cells. Cell 114: 585-598, 2003.
8. Seki A, Coppinger JA, Jang CY, Yates JR and Fang G: Bora and the kinase Aurora a cooperatively activate the kinase Plk1 and control mitotic entry. Science 320: 1655-1658, 2008.

9. Macurek L, Lindqvist A, Lim D, Lampson MA, Klompmaker R, Freire R, Clouin C, Taylor SS, Yaffe MB and Medema RH: Polo-like kinase-1 is activated by aurora A to promote checkpoint recovery. Nature 455: 119-123, 2008.

10. Macurek L, Lindqvist A and Medema RH : Aurora-A and hBora join the game of Polo. Cancer Res 69: 4555-4558, 2009.

11. Hong YR, Chen CH, Chang JH, Wang S, Sy WD, Chou CK and Howng SL: Cloning and characterization of a novel human ninein protein that interacts with the glycogen synthase kinase 3beta. Biochim Biophys Acta 1492: 513-516, 2000.

12. Howng SL, Hsu HC, Cheng TS, Lee YL, Chang LK, Lu PJ and Hong YR: A novel ninein-interaction protein, CGI-99, blocks ninein phosphorylation by GSK3beta and is highly expressed in brain tumors. FEBS Lett 566: 162-168, 2004.

13. Dar AA, Belkhiri A and El-Rifai W: The aurora kinase A regulates GSK-3beta in gastric cancer cells. Oncogene 28: 866-875, 2009.

14. Fields S and Song O: A novel genetic system to detect proteinprotein interactions. Nature 340: 245-246, 1989.

15. Zhu L: Yeast GAL4 two-hybrid system. A genetic system to identify proteins that interact with a target protein. Methods Mol Biol 63: 173-196, 1997.

16. Cheng TS, Hsiao YL, Lin CC, et al: Glycogen synthase kinase 3 beta interacts with and phosphorylates the spindle-associated protein astrin. J Biol Chem 283: 2454-2464, 2008.

17. Elbashir SM, Harborth J, Lendeckel W, Yalcin A, Weber K and Tuschl T: Duplexes of 21-nucleotide RNAs mediate RNA interference in cultured mammalian cells. Nature 411: 494-498, 2001.

18. Harborth J, Elbashir SM, Bechert K, Tuschl T and Weber K: Identification of essential genes in cultured mammalian cells using small interfering RNAs. J Cell Sci 24: 4557-4565, 2001.

19. Church GM and Gilbert W: Genomic sequencing. Proc Natl Acad Sci USA 81: 1991-1995, 1984.

20. Matsuoka S, Ballif BA, Smogorzewska A, et al: ATM and ATR substrate analysis reveals extensive protein networks responsive to DNA damage. Science 316: 1160-1166, 2007.

21. Kufer TA, Silljé HH, Körner R, Gruss OJ, Meraldi P and Nigg EA: Human TPX2 is required for targeting Aurora-A kinase to the spindle. J Cell Biol 158: 617-623, 2002. 